\title{
On the hysteretic operators with random parameters
}

\author{
Mikhail E. Semenov ${ }^{1,2,3,4, *}$, Olesia I. Kanishcheva ${ }^{2}$, Peter A. Meleshenko ${ }^{1,2, * *}$, Olga O. Reshetova ${ }^{1}$, Roman E. \\ Pervezentzev $^{2}$, and Alexander $V$. Budanov ${ }^{5}$ \\ ${ }^{1}$ Voronezh State University, Universitetskaya sq. 1, 394006, Voronezh, Russia \\ ${ }^{2}$ Zhukovsky-Gagarin Air Force Academy, Starykh Bolshevikov st. 54 "A", 394064, Voronezh, Russia \\ ${ }^{3}$ Voronezh State Technical University, XX-letiya Oktyabrya st. 84, 394006 Voronezh, Russia \\ ${ }^{4}$ Geophysical Survey of Russia Academy of Sciences, Lenina av. 189, 249035 Obninsk, Russia \\ ${ }^{5}$ Voronezh State University of Engineering Technology, Revolution av. 19, 394036 Voronezh, Russia
}

\begin{abstract}
In this work we introduce the novel class of hysteretic operators with random parameters. We consider the definition of these operators in terms of the "input-output" relations, namely: for all permissible continuous inputs corresponds the output in the form of stochastic Markovian process. The properties of such operators are also considered and discussed on the example of a non-ideal relay with random parameters. Application of hysteretic operators with stochastic parameters is demonstrated on the example of simple oscillating system and the results of numerical simulations are presented.
\end{abstract}

\section{Introduction}

Hysteresis phenomena are of interest both from the fundamental and applied points of view. Such an interest is caused by high incidence of these phenomena in a various technical systems (such as robotic, mechanical, electromechanical systems, management systems for tracking of aircrafts etc.) Also, these phenomena determine some unusual elasto-plastic properties of modern nanomaterials (such a properties are served as a base for construction of modern self-healing materials) on the basis of fullerene films. Moreover, the hysteretic phenomena are widely known in biology, chemistry, economics, etc. It should be noted that the hysteretic behavior of such systems is caused by either their internal structure, or the presence of separate blocks with hysteretic characteristics. Of course, when modeling the dynamics of such systems, it is necessary to use an adequate mathematical apparatus.

Currently used models of hysteretic phenomena both constructive (such as non-ideal relay, Preisach and Ishlinskii-Prandtl models, etc. [1]), and phenomenological (Bouc-Wen model, Duhem model, etc. [2]) assume a priori the stability of the parameters that identify the hysteretic properties of the corresponding operators. However, the stability of parameters in real engineering systems (e.g., in the systems modelled by the coupled inverted pendula [3]) does not always take place. In this way, such operators are the natural model in the situation when the parameters of hysteresis carrier are under influence of stochastic, uncontrollable affections. For example, it is difficult to control the switching numbers of non-ideal relay, which is a part of control systems of the corresponding de-

\footnotetext{
*e-mail: mk1150@mail.ru

**e-mail: melechp@yandex.ru
}

vices, when the various external factors take place (in this case the switching numbers may be subjected to random changes). These circumstances make it necessary to develop the extended models of hysteretic effects, taking into account the stochastic changes of the parameters that are deteremined the corresponding hysteretic operators. We note that the equations with random parameters (principally such equations are linear) were considered in [4-6]. The strongly nonlinear differential equations containing the operator nonlinearity with random properties have not been considered in the literature. Thus, construction and investigation of the properties of hysteretic operators with random parameters seems novel and promising problem.

\section{Non-ideal relay with random parameters}

Let us consider a non-ideal relay (a detailed description of this and other hysteretic converters, as well as their properties in the case of deterministic parameters, are given in the classical book of M.A. Krasnosel'skii and A.V. Pokrovskii [7]), in which the switching numbers are not fixed, but are treated as random variables with absolutely continuous distribution function. Concerning these random variables, we make the following assumption: the probability density of each of the switching numbers will be assumed to be finite with non-intersecting supports. We denote these switching numbers as $\varphi_{\alpha}(u)$ and $\varphi_{\beta}(u)$. We will consider the case when the supports of the function $\varphi_{\alpha}(u)$ and $\varphi_{\beta}(u)$ are contained in the intervals $\left[u_{\alpha}^{-}, u_{\alpha}^{+}\right]$and $\left[u_{\beta}^{-}, u_{\beta}^{+}\right]$, respectively.

Following the basic ideas presented in [7], the dynamics of the input-output relations for the operator of a nonideal relay with random switching numbers is determined by two relations, namely: "input-state", "state-output". 
We assume that all permissible for the converter $R$ continuous inputs are given on the non-negative semi-axis $(t>0)$ (the input-output relation for this converter has the form $\left.x(t)=R\left[t_{0}, x_{0}, \alpha, \beta\right] u(t),\left(t \geq t_{0}\right)\right)$. The space of possible states of such an operator is defined as $\Omega=\Omega(\omega, p, u)$, $(\omega=0,1,0 \leq p \leq 1,-\infty<u<+\infty)$.

The variable state of the converter $R\left[\left(1 ; p_{0}\right) ; x_{0} ; \varphi_{\alpha}(u) ; \varphi_{\beta}(u)\right] u(t)$ is a random value that takes the value 0 with probability $(1-p(t))$ and a value of 1 with probability $p(t)$. In other words it can be presented as a pair $\{1 ; p(t)\}$ (here the second output component corresponds to the probability that at the time $t$ the first component is 1). The output of this converter is a random function $x(t)$ (Markovian process) taking a value of 1 with probability $p(t)$. The rule that determines the value of probability $p(t)$ will be given below.

\subsection{Definition of input-output relation for a non-ideal relay with random switching numbers}

Following the classical scheme proposed in [7], we give the definition of the input-output relation by means of a three-step construction:

- At the first step we define the input-output relation on the monotonic inputs only;

- At the second step, using the semi-group identity, the input-output relation is defined for all piece-wise monotonic inputs;

- At the third step, using the special limit construction, the corresponding converter will be defined for all monotonic inputs.

We define the operator $R$ on the monotonic inputs. Let us assume that at the initial time point $t_{0}$ (to simplify the calculations, we assume that $t_{0}=0$ ) the operator $R$ is in the state $1 ; p_{0} ; u_{0} \in \Omega,\left(u(0)=u_{0}\right)$. Let the input $u(t)$ be a monotonic increase, then for the time $t>0$ the output is $x(t)=\{1 ; p(t)\}$ where

$$
p(t)=\max \left\{p_{0} ; \int_{-\infty}^{u(t)} \varphi_{\beta}(u) d u\right\} .
$$

The semi-group identity for the operator $R$ immediately follows from the definition. Let $t_{1}$ be an arbitrary moment of time satisfying the inequality $0<t_{1}<t$, then the semi-group identity for the operator of a non-ideal relay has the form:

$$
\begin{aligned}
& R\left[t_{0} ; p_{0} ; u_{0} ; \varphi_{\alpha} ; \varphi_{\beta}\right] u(t)= \\
& =R\left[t_{1} ; R\left[t_{0} ; p_{0} ; u_{0} ; \varphi_{\alpha} ; \varphi_{\beta}\right] u\left(t_{1}\right) ; u\left(t_{1}\right) ; \varphi_{\alpha} ; \varphi_{\beta}\right] u(t)
\end{aligned}
$$

To define an operator on the piece-wise monotonic inputs (in the case of a finite interval $[0, T]$ ), we break this interval by points $t_{1}, t_{2}, \ldots, t_{n}$ in to intervals of monotonicity. On each of them we define the corresponding operator as an operator on a strictly monotonic input whose initial state will be defined as the state at the instant corresponding to the "last" change in the behavior of the input.
To determine the operator $R$ on continuous inputs, we use the following limit construction. Let $u(t)(t \in[0, T])$ be an arbitrary continuous input. Let us consider an arbitrary sequence of piece-wise monotonic inputs $u_{n}(t),(n=$ $1,2, \ldots)$ that converges uniformly to each element of this sequence $u(t)$. A single-variable state $p_{n}(t),(n=1,2, \ldots)$ will form a sequence of state variables $p_{n}(t),(n=1,2, \ldots)$. Let us prove that the sequence $p_{n}(t),(n=1,2, \ldots)$ converges uniformly. We estimate the absolute value of the difference:

$$
\begin{aligned}
& \left|p_{n}(t)-p_{m}(t)\right| \leq \max _{t}\left|\int_{-\infty}^{u_{n}(t)} \varphi_{\alpha}(u) d u-\int_{-\infty}^{u_{m}(t)} \varphi_{\alpha}(u) d u\right|= \\
& =\max _{t}\left|\int_{u_{n}(t}^{u_{m}(t)} \varphi_{\alpha}(u) d u\right| .
\end{aligned}
$$

Since the function $\varphi_{\alpha}(u)$ is continuous, and because of uniform convergence also

$$
\lim _{n, m \rightarrow \infty} \max _{t}\left|u_{n}(t)-u_{m}(t)\right|=0,
$$

as well as, using the mean value theorem:

$$
\max _{t}\left|\int_{u_{n}(t}^{u_{m}(t)} \varphi_{\alpha}(u) d u\right| \leq \max _{t} \varphi_{\alpha}(t)\left[u_{n}(t)-u_{m}(t)\right]
$$

the right-hand side of the inequality (3) tends to zero. Thus, the sequence of probabilities $p_{n}(t)$ is fundamental (the continuity is obvious), then there is $\lim _{n \rightarrow \infty} p_{n}(t)=p(t)$, which is comparable to an arbitrary continuous input $u(t)$.

\subsection{Monotonicity of a non-ideal relay with random parameters}

Let us consider the monotonicity property for the constructed converter. We determine the monotonicity with respect to the initial state of the non-ideal relay: if $\left\{u\left(t_{0}, x_{0}\right\},\left\{v\left(t_{0}, y_{0}\right\} \in \Omega(\alpha, \beta), x_{0} \leq y_{0}\right.\right.$ and $u(t) \leq v(t)(t \geq$ $\left.t_{0}\right)$, then $R\left[t_{0}, x_{0}, \alpha, \beta\right] u(t) \leq R\left[t_{0}, x_{0}, \alpha, \beta\right] v(t)\left(t \geq t_{0}\right)$.

Let us note that this property can be used as the definition of a non-ideal relay. In order to use it, we define the outputs corresponding to monotonic inputs. Applying a semi-group identity, we define the outputs for piecewise monotonic inputs. Further, this relation is extended by means of the special limit construction to all monotonic inputs. In this case, this relation will be the exclusive. We can also note a natural monotonicity in the switching numbers. With respect to the modified operator of a non-ideal relay with random parameters, the analogue of monotonicity can be presented in the form of the following theorem.

Theorem 1. Let $p\left\{x_{01}=1\right\} \geq p\left\{x_{02}=1\right\}$ and $x_{1}(t) \geq$ $x_{2}(t)$. Then for any $t: p\left\{x_{1}=1\right\} \geq p\left\{x_{2}=1\right\}$.

\section{Dynamics of a system under non-ideal relay with random parameters}

In order to show the action of the developed operator on the real physical system let us consider the simple oscillating system under hysteretic force with random parameters. 
Such a simple system is considered in $[8,9]$ and the external force has the form of a non-ideal relay with inversion of the switching numbers. One of the main results of these studies is the existence of unlimited solutions, namely, if the initial conditions are such that the hysteretic operator is triggered at the first cycle of oscillations, then the corresponding solution is unlimited, while the growth rate of the amplitude is proportional to the square root of time. Let us consider an analogous system with a non-ideal relay with switching numbers distributed according to an evendimensional law. The equation of motion together with the corresponding initial conditions has the following form:

$$
\begin{aligned}
\ddot{x}(t)+\omega^{2} x(t) & =R\left[t_{0} ; p_{0} ; u_{0} ; \varphi_{\alpha} ; \varphi_{\beta}\right] x(t), \\
x(0) & =x_{0}, \dot{x}(0)=x_{1} .
\end{aligned}
$$

To implement the numerical solution of the system (4), it is necessary to generate a set of random values corresponding to the switching numbers. Let us consider the case when $\varphi_{\alpha}(u)$ and $\varphi_{\beta}(u)$ corresponds to the uniform distribution law for $\alpha$ and $\beta$. For definiteness, we will assume that these functions correspond to uniform distributions in the intervals $[-1.5,-0.5]$ and $[0.5,1.5]$, respectively. The solution of the system (4) can be obtained by specifying the initial conditions and the values of the switching numbers corresponding to the non-ideal relay. At each period, the switching numbers are selected from the corresponding distributions with the initial conditions for the next realization corresponding to the values of the phase coordinates obtained at the previous step. Using the described algorithm, a solution to the system (4) is obtained and the corresponding law of motion together with the phase portrait are shown in Fig. 1.
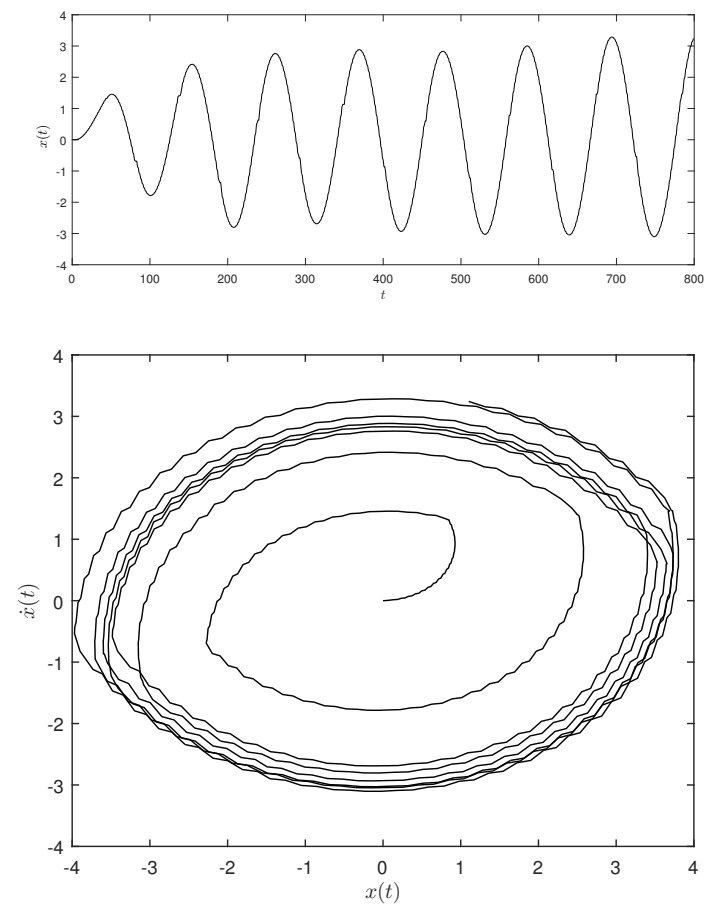

Figure 1. Solution (top panel) and phase portrait (bottom panel) for the system (4)
The following theorem characterizes the dynamics of the system (4).

Theorem 2. Let us suppose that the supports of the function $\varphi_{\alpha}$ and $\varphi_{\beta}$ do not intersect. Then $\overline{\lim _{t \rightarrow \infty} x(t)}=\infty$, that is the amplitude tends to infinity with probability 1 .

The proof of this theorem follows from the fact that the area of the minimal hysteretic loop is positive $S_{\min }>0$, as a consequence, the amplitude value at each cycle satisfies the inequality:

$$
A_{n}^{2}(t) \geq n S_{\min }
$$

We also note that under the conditions of the theorem, the rate of growth of the amplitude with probability 1 is proportional to the square root of time.

\section{Conclusions}

The paper presents a generalization of the classical hysteretic converter in the form of non-ideal relay to the case when its switching numbers are randomly distributed according to a corresponding law. The properties of this converter are established (namely, the definition, together with the monotonicity), as well as the dynamics of the simple mechanical system in the form of oscillator under hysteretic force determined by a non-ideal relay with random parameters is considered.

This work is supported by the RFBR (Grants 16-08-00312-a, 17-01-00251-a, and 18-08-00053-a)

\section{References}

[1] M.E. Semenov et al., MATEC Web of Conference 83, 01008(1)-01008(5) (2016)

[2] F. Ikhouane, V. Mañosa, J. Rodellar, Systems \& Control Letters 56, 197-205 (2007)

[3] M.E. Semenov et al., Archieve of Applied Mechanics 88, 517-524 (2018)

[4] Øksendal Bernt, Stochastic Differential Equations. An Introduction With Applications (Berlin, SpringerVerlag, 2003) $379 \mathrm{p}$

[5] V.G. Zadorozhniy, Computational Mathematics and Mathematical Physics 53, 486-502 (2013)

[6] V.G. Zadorozhniy, S.S. Khrebtova, Computational Mathematics and Mathematical Physics 49, 18531868 (2009)

[7] Krasnosel'skii M.A., Pokrovskii A.V., Systems with Hysteresis (Berlin, Springer-Verlag, 1989) $410 \mathrm{p}$

[8] A.M. Solovyov et al., Procedia Engineering 201, 549-555 (2017)

[9] A.M. Solovyov et al., Procedia Engineering 201, 578-583 (2017) 